\title{
The role of Satellite Communications in the Smart Grid
}

\author{
Alessio Meloni and Luigi Atzori \\ DIEE - Department of Electrical and Electronic Engineering \\ University of Cagliari \\ Piazza D’Armi, 09123 Cagliari, Italy \\ Email: $\{$ alessio.meloni $\}\{1$. atzori $\} @$ diee.unica.it
}

\begin{abstract}
The dramatic drop in the price of equipment and services and the increase in efficiency candidate Satellite Communications (SATCOM) as an interesting choice for the deployment of major Smart Grid scenarios. As a matter of fact, SATCOM providers are offering more and more services dedicated to machine-to-machine communications and they expect actors in the smart grid domain to be among the main users. However, to fruitfully integrate this communication technologies into smart grids, it is of utmost importance to understand strengths and limits of the available satellite features in light of smart grid communication requirements and recent advancements in the SATCOM arena. Starting with a review on the latest trends in the satellite market for machine-to-machine communications, relevant smart grid requirements are classified and mapped to the most significant smart grid applications. Then, various features and trends of SATCOM are discussed to take stock of their suitability and to outline future challenges for the exploitation of SATCOM systems in smart grids.
\end{abstract}

Index Terms-Smart Grid, Satellite Communications.

\section{INTRODUCTION}

The electric grid is currently facing massive changes in light of a combination of interrelated technical, political and economic drives. The most important of these changes deals with making the grid smarter by enabling the communication of relevant information across the entire network. This will help towards the creation of new services and applications with the goal of a more efficient, reliable, secure and cost-effective system in all the phases from generation to consumption.

A significant quantity of new data are being produced by new intelligent devices such as Smart Meters (SMs) and Phasor Measurement Units (PMUs). These will add to those already exchanged in the Smart Grid (SG) by means of Supervisory Control And Data Acquisition (SCADA). In addition, also cross-domain actors such as Electric Vehicles (EVs) will play an important role joining the SG information pool.

In order to really leverage on the advantages brought by all these data producers, it is extremely important to build an information and communication overlay which is able to support the requirements dictated by the case studies while taking into account the impact of future SG data on the

(C)2017 IEEE. Accepted for publication in IEEE Wireless Communications. This is a preprint version. For the final version, please visit: http://ieeexplore.iee.org/abstract/document/7909157. The IEEE copyright notice applies. DOI: 10.1109/MWC.2017.1600251 overall communication network. For this reason, the future ICT infrastructure for the SG must consider any viable technology including those that have been overlooked in the past as satellite communications (SATCOM).

SATCOM can provide extensive coverage and rapid installation and deployment in those scenarios in which terrestrial communication infrastructures either do not exist, are too costly or are not sufficient for the domain-specific requirements. In the past, the use of SATCOM in power systems was limited to SCADA because of the insufficient performance and high costs. Now, the role that SATCOM can play in the SG domain must be rediscussed in light of the improved performance of SATCOM coupled with the reduction of costs and the Quality of Service (QoS) requirements expected by new SG applications. Indeed, it has been forecasted that, by 2020 , the revenue from satellite services and equipment dedicated to SG applications will total $\$ 368$ million [1]. Based on these considerations, this paper gives a thorough overview on how to fruitfully integrate SATCOMs into SG overlay networks to understand the role SATCOMs can play in different application scenarios. To ease the reading of the paper, a list of abbreviations is provided at the end of the paper.

\section{RECENT TRENDS OF SATCOM TOWARDS M2M APPLICATIONS}

While in the past the primary commercial use of SATCOM was in one-way communications for media services, nowadays two-way communication systems such as those providing IP services for machine-to-machine (M2M) applications are increasingly becoming common [2]. Also, over the years ground terminal dishes have become smaller and satellite technologies have continuously advanced, making the use of SATCOM more efficient and less expensive. In the past, data rates of few kbps were considered cutting-edge satellite technology for the provision of IP services. Today, data rates up to few Mbps are possible with compact equipment.

As a matter of fact, global leaders of SATCOM systems have started to provide services specifically intended for M2M. Inmarsat has launched Broadband Global Area Network (BGAN) M2M, which can reach a maximum data rate of nearly $500 \mathrm{kbps}$, latencies below $1 \mathrm{~s}$ and availability of the service of $99.9 \%$. Iridium has a network of 66 satellites 
covering the whole earth with low latencies due to the low orbit of its satellites and resilience to weather conditions due to the use of the L-band. Moreover, a new constellation of satellites called Iridium NEXT will be active from 2017 in order to offer data rates up to $1.4 \mathrm{Mbps}$. Also companies specialized in M2M over satellite are becoming popular. The most famous is Orbcomm, which has recently launched a nextgeneration satellite constellation called $O G 2$ which is the first network entirely devoted to M2M.

Given the needs and the recent trends in SATCOM towards M2M applications, the role of this communication technology in the fast-evolving scenario of SG needs to be discussed by identifying domain-specific requirements, key features and criticalities. [3] gives analytical tools to measure the feasibility of satellite systems with different orbit configurations into M2M applications. [2] provides an overview on how satellite communications can support remote communications in SG, environmental monitoring and emergency management applications. In [4], the feasibility of IP data services over satellite for supporting active network management solutions in distribution networks was explored. The goal of this paper is to give a reference and stimulate further insights and studies on the role that SATCOM can play in the SG.

\section{QoS REQUiREMENTS OF SG APPLICATIONS}

Table I: QoS requirements for SG applications.

\begin{tabular}{|c|c|c|c|}
\hline $\begin{array}{c}\text { QoS } \\
\text { requirements }\end{array}$ & Low & Moderate & High \\
\hline Latency & $>1 \mathrm{~s}$ & $\begin{array}{c}100 \mathrm{~ms}-1 \mathrm{~s} \\
\text { (near real time) }\end{array}$ & $\begin{array}{c}\leq 100 \mathrm{~ms} \\
\text { (real time) }\end{array}$ \\
\hline Availability & $<99 \%$ & $99-99.99 \%$ & $>99.99 \%$ \\
\hline Bandwidth & $9.6-56 \mathrm{kbps}$ & $56-100 \mathrm{kbps}$ & $>100 \mathrm{kbps}$ \\
\hline Activity & $\begin{array}{c}\text { infrequent \& } \\
\text { deterministic }\end{array}$ & $\begin{array}{c}\text { frequent \& } \\
\text { deterministic }\end{array}$ & variable \\
\hline Mobility & $\begin{array}{c}\text { fixed } \\
\text { terminals }\end{array}$ & $\begin{array}{c}\text { both fixed and } \\
\text { mobile terminals }\end{array}$ & $\begin{array}{c}\text { mobile } \\
\text { terminals }\end{array}$ \\
\hline
\end{tabular}

Table I shows the major QoS requirements of SG applications, as analyzed by both standardization [5] and research [6] communities. Three levels are possible for each parameter: High $(H)$, Moderate $(M)$ and Low $(L)$.

With reference to the latency, the possible scenarios range from outage-critical applications that require real time communications and always-on availability (e.g., substation automation) to applications which are not fail-critical for the power system but help in optimizing its management (e.g., smart metering) [5].

The most critical SG applications have also stringent requirements in terms of availability of the communication system. For example, an availability above $99.99 \%$ means that the communication system cannot be down for more than 50

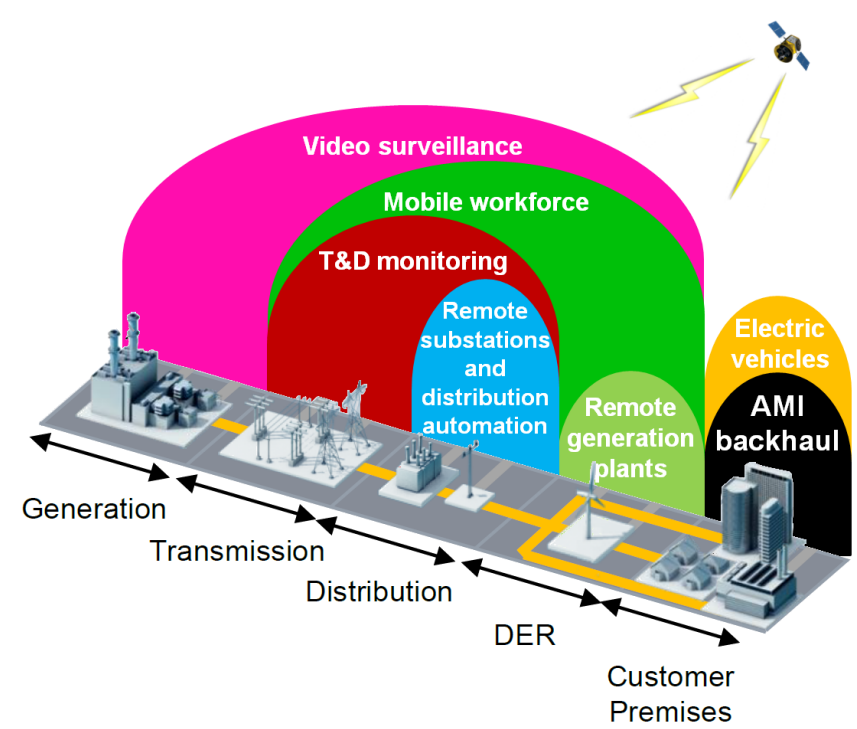

Figure 1: SG applications and domains involved.

minutes per year, which can still be critical for some protection applications requiring utmost reliability.

As to the activity, each SG application is classified based on the interval between adjacent data transmissions and whether these intervals are variable or deterministic [7]. In some cases, infrequent but deterministic (i.e., periodic) communications are required, which are then easy to schedule as it is the case of billing automation. Some other applications need frequent (i.e., with intervals no more than a few seconds long) and deterministic (i.e., constant) transmissions which are also easy to schedule but introduce some challenge when more applications share the same channel. Finally, some applications require communications classified as variable, which means they are not schedulable and work according to the actual needs. Variable activity applications are clearly the most challenging. Whether variable activity can be considered frequent or not, depends on many factors and can change even for the same SG application.

The need of bandwidth by SG applications is increasing at a fast pace. While in the past narrowband communications for SCADA systems represented the most common scenario, nowadays SG applications requiring higher data rates exist, which are usually divided among those below and above 100 kbps [6].

As to the mobility, most of the SG applications deal with fixed terminals. However, for the few applications requiring mobility, this aspect should not be overlooked since some satellite features deeply affect the QoS in mobile scenarios.

Concerning security, no classification is given since any SG application has high requirements, as highlighted in [6]. In general, SATCOM are considered a safer and harder to disrupt technology than its terrestrial counterparts [3]. Nevertheless, eavesdrop threats in the case of large satellite footprints should be taken into account. 


\section{SG APPLICATIONS EXPLOITING SATCOM}

SATCOM can play a very important role in the SG domain, e.g., in rural areas poorly served by other communication technologies and as a land infrastructure-independent backup connection. Figure 1 shows a plethora of SG applications which can exploit SATCOM, mapping them to the domains where related devices are installed. The following sections present the classification of the QoS requirements for major applications, which is illustrated in Figure 2.

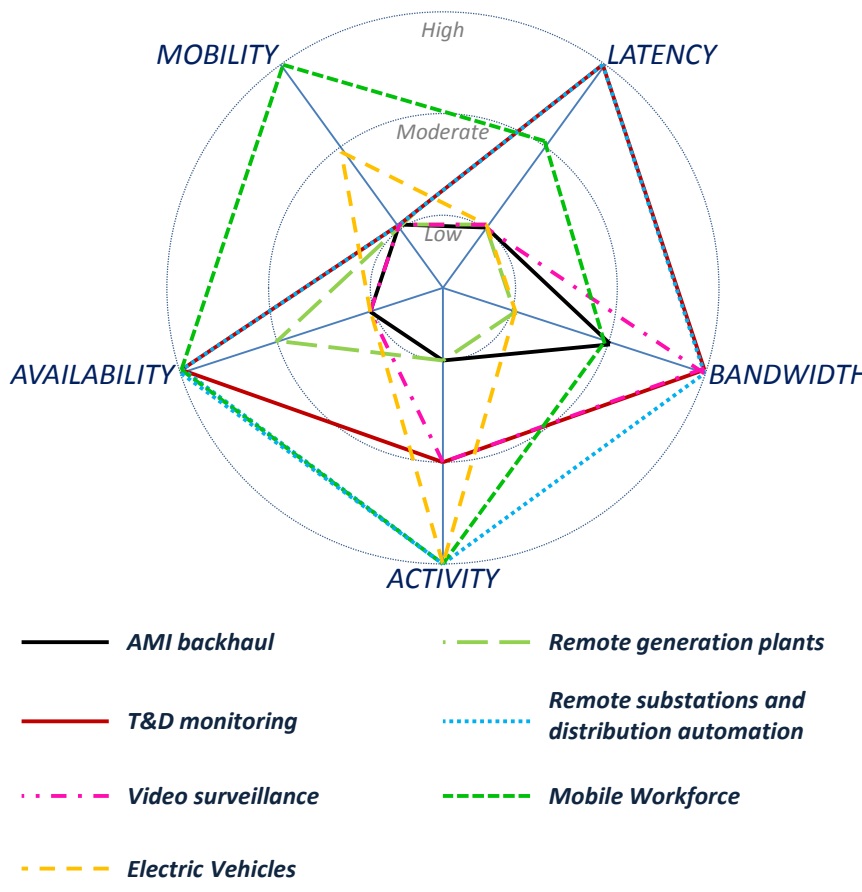

Figure 2: Mapping of QoS requirements for major SG applications

\section{A. Advanced Metering Infrastructure (AMI) backhaul}

SMs data are useful for many purposes, such as billing and demand-response. Due to the cost of a SATCOM terminal and the limited amount of data to be sent in this scenario, a single gateway node to send meters data is often used for a group of houses. Currently, different points of view about the reporting period of SMs exist. The most agreed upon is 15 minutes which is enough to accommodate demand-response programs [6]. However, for the future, utilities are willing to use smaller periods to further optimize the management of the electrical network and to more promptly react to events happening in the grid. Latency requirements are assumed low [6]. Also, availability is not a critical requirement for most of the services using smart meters data. Nevertheless, in some cases pseudomeasurements (i.e., estimated data) can be used by utilities. As to the bandwidth allocated, the requirements vary depending on how many SM's flows are aggregated by the gateway. Typical packet sizes for SMs are approximately 200 Bytes while feeders usually serve 250 to 500 customers [7]. Therefore, the relevant requirement typically falls under the moderate category.

\section{B. Remote generation plants}

Currently, more and more Distributed Energy Resources (DER) of various sizes are being built to exploit the available renewable sources. In this case, the aim is both to monitor the status of the generation plant for operational purposes and to know the production to match the energy offer and demand. Similarly to AMI backhaul, low latency requirements are assumed while availability requirements are moderate because of the role played in the overall balance of the grid. Bandwidth requirements are low because differently from the previous application, the terminal does not aggregate multiple sources of data. Also activity is low as in the case of AMI backhaul, although shorter intervals are likely to be used in the future for operational purposes.

\section{Transmission and Distribution $(T \& D)$ monitoring}

$T \& D$ networks are increasingly being equipped with powerful intelligent sensing devices which help monitoring and estimating the state of the system at any given time over a wide area. The most famous example are PMUs, which are able to measure voltage, current, frequency and other quantities with continuous rates as high as 120 times per second. Typical sizes of a measurement packet using C37.118.2-2011 standard (which is IP-compliant) are in the order of hundreds of Bytes [7]. Therefore, the activity requirement is moderate while the bandwidth requirement is high. Latency requirements are generally assumed high because of the role $T \& D$ can play in many critical situations. For the same reason, high availability is expected.

\section{Remote Substations and Distribution Automation}

Distribution lines connect transmission lines to endcustomers via distribution feeders. Substations transform the voltage levels according to the needs of the T\&D lines and are composed of many devices and functions to provide safety and stability of the system. Thus, substations must be equipped with remote communication so that control and decisions upon these critical operations can be taken from remote. IEC 61850 is currently the protocol chosen for this task and it is designed to run over communication networks using the IP standard [5]. However, also DNP-3 can be found in older SCADA systems. The expected latency and availability requirements are high in light of the crucial role performed. The bandwidth requirement are assumed to be high because of the number of devices to pilot [7]. Activity requirements are high, since the number of control communications strictly depends on the state of the electric network.

\section{E. Video surveillance of remote sites}

Video surveillance of remote sites is not a new SG application. Utilities already utilize SATCOM to monitor remote assets. Nevertheless, this is one of the most expensive and bandwidth consuming application since it requires continuous transmission of data. In this case, latency requirements are low since the application does not have any constraint on actions to take promptly (i.e. below $1 \mathrm{~s}$ ). Also percentages of availability 
under 99\% are acceptable. In case the communication channel is not available, data can be buffered and sent as soon as possible in a best effort manner.

\section{F. Mobile Workforce}

Mobile workforce networks are used by utilities to provide dispatch and maintenance. Satellite systems can provide their contribution especially for in-vehicle use in blind areas. Workforce vehicles can be equipped with satellite equipment or handheld devices can be used, so that communication can be provided in case cellular services or other wireless technologies are not available, i.e. in hard-to-reach areas. Delays below $200 \mathrm{~ms}$ are usually targeted for calls over satellite although latencies up to $1 \mathrm{~s}$ are still acceptable. Also, setup delays above $1 \mathrm{~s}$ are acceptable when beginning a call. The bandwidth necessary usually does not exceed $100 \mathrm{kbps}$ while the availability must be high during calls, which of course have variable activity. Last, differently from the other applications presented so far, mobility requirements are high and should be taken into account when choosing the best satellite features.

\section{G. Electric Vehicles}

The market of EVs is annually growing at rates above $20 \%$. EVs can add value to the optimization of the SG, since they can be seen both as loads and sources for power supply. However, they also represent an additional energy demand which will impact the SG. Therefore, knowing the exact location of EVs, their battery charge but also owners' related information such as when the car is parked and when it will be used next, are important information for the optimization of the SG. In the future, EVs will be able to communicate among themselves and with Road Side Units (RSUs) providing remote connectivity [8]. Especially when EVs are far from urban centers, the use of SATCOM could be the best solution for keeping track of the EVs related information both with direct communications of the EVs over satellite or by means of satellites connected to RSUs. The requirements expected on latency, availability and bandwidth are low while the activity requirements are high because of the variability of communications expected.

\section{Suitability of SATCOM FeATURES TO SMART GRID REQUIREMENTS}

Key features characterize SATCOM communications, which are analyzed in the following to better understand their impact on deployed SG applications. These features are listed in Table II together with the preferred configurations per application, as it arises from the following sections.

\section{A. Orbits}

Orbits [9] are the most intrinsic and distinguished feature of SATCOM that deeply affect the latency, availability, bandwidth and mobility of the terminals: Geostationary Earth Orbit (GEO) satellites float at an altitude of approximately $36000 \mathrm{~km}$ and appear stationary from the earth surface; Medium Earth
Orbit (MEO) satellites range from 2000 to $35000 \mathrm{~km}$ and can have an orbital revolution as long as 10 hours; Low Earth Orbit (LEO) systems range from 160 to $2000 \mathrm{~km}$ of altitude and have the shortest revolution period.

The distance of the satellite from the earth affects the bandwidth and the availability because of the relative footprint and the pointing accuracy needed: the farther the satellite, the higher the pointing accuracy needed. Accordingly, LEO's are the most permissive, making them attractive for the use with EVs and mobile workforce. On the other hand, MEO systems require expensive terminal equipment able to continuously change the orientation of the antenna. This is not required for GEO systems, as these are used with fixed terminals, for applications such as video surveillance, AMI backhaul and remote generation plants, which can benefit from a large footprint to gather data from wide areas without disruptions caused by satellite handovers. Differently, in MEO and LEO systems, a constellation made of many different satellites is compulsory to have a reliable service.

Another important influence of the satellite altitude is over the propagation time: the Round Trip Time (RTT) in GEO is at least $500 \mathrm{~ms}$ while in MEO and LEO can be as low as few tens of ms and few ms respectively. Therefore, critical services such as T\&D monitoring and distribution automation should use LEO, although a constellation providing a reliable service is extremely important. MEO is typically used for navigation systems and for communication scenarios in which it is important to have a larger footprint than the one LEO guarantees, notwithstanding the cost and the complexity. Such an example are satellite systems providing communication coverage to the earth poles.

Recently, interest in the use of small satellites called nanosatellites which operate in LEO increased. Nanosatellites can reach data rates of up to $9.6 \mathrm{kbps}$ and have small production and launch costs, but they present many challenges in the setup of the communication link due to orbit constraints imposed by their power, mass and volume. Apart from the low data rate, the limited contact time with each nanosatellite yields to intermittent and disruptive communication links, making it difficult to appropriately plan the coverage of a given area. For these reasons, research on how to improve the reliability is ongoing. At the moment, nanosatellites represent an interesting technology only for delay tolerant applications requiring small data rates and availability, such as remote generation plants.

Summarizing, GEO and LEO are the most interesting for most SG applications. More information on the differences between them can be found in [3]. Currently, M2M providers are concentrating more on LEO for two reasons: the GEO belt is overcrowded and battery-powered terminals using LEO systems drain less power because of the smaller distance. Recently, a successful demonstration of Long Range Wide Area Network (LoRaWAN) has been accomplished by Stream Technologies and Global Satellite Engineering over the Iridium network made of LEO satellites, showing the advantages of LEO systems in battery-powered devices using SATCOM. Indeed, LoRaWAN is intended for low power wide area networks accessed by battery operated devices over wireless 
Table II: SATCOM features and technologies and suggested use for the presented SG applications ("+" means recommended, "o" means generally suited, but not first priority, “-” means not recommended).

\begin{tabular}{|c|c|c|c|c|c|c|c|c|c|}
\hline Key feature & $\begin{array}{c}\text { Sub } \\
\text { feature }\end{array}$ & $\begin{array}{c}\text { Key differentiating } \\
\text { properties }\end{array}$ & $\begin{array}{c}\text { AMI } \\
\text { backhaul }\end{array}$ & $\begin{array}{c}\text { Remote } \\
\text { generation } \\
\text { plants }\end{array}$ & $\begin{array}{c}\text { T\&D } \\
\text { monitoring }\end{array}$ & $\begin{array}{c}\text { Remote } \\
\text { substations } \\
\text { and } \\
\text { distribution } \\
\text { automation }\end{array}$ & $\begin{array}{c}\text { Video } \\
\text { surveillance }\end{array}$ & $\begin{array}{c}\text { Mobile } \\
\text { Workforce }\end{array}$ & $\begin{array}{l}\text { Electric } \\
\text { vehicles }\end{array}$ \\
\hline \multirow{4}{*}{ Orbits } & GEO & Large and stationary footprint & + & + & o & o & + & - & - \\
\hline & MEO & Medium footprint and delay & o & o & o & o & o & - & - \\
\hline & LEO & Lower delay and pointing req. & o & o & + & + & o & + & + \\
\hline & Nanosatellites & Cheap but poorly reliable & o & + & o & - & - & o & o \\
\hline \multirow{4}{*}{$\begin{array}{l}\text { Physical } \\
\text { layer } \\
\text { features }\end{array}$} & Low bands & Lower data rate, more reliable & o & o & o & o & - & + & + \\
\hline & High bands & Higher data rate, less reliable & + & + & o & o & + & - & - \\
\hline & Dual band & Best reliability but expensive & o & o & + & + & - & o & - \\
\hline & $\mathrm{ACM}$ & Adaptive to weather & o & o & o & o & + & + & o \\
\hline \multirow{3}{*}{$\begin{array}{c}\text { Channel } \\
\text { Management }\end{array}$} & FA & QoS guarantee & o & o & + & + & + & - & - \\
\hline & DAMA & Variable traffic, high data rate & + & o & o & o & o & + & o \\
\hline & RA & Best effort & - & + & - & - & - & o & + \\
\hline \multirow{2}{*}{$\begin{array}{c}\text { Local } \\
\text { Intelligence }\end{array}$} & Inwards & Reduced latency & o & o & o & + & - & - & - \\
\hline & Outwards & Bandwidth and power saving & + & + & + & + & + & o & o \\
\hline \multirow{3}{*}{$\begin{array}{c}\text { Open } \\
\text { networking } \\
\text { and transport } \\
\text { protocols }\end{array}$} & LTE & Open and interoperable & - & o & - & o & o & + & o \\
\hline & DVB & IPv6 compliant & + & + & + & + & + & o & + \\
\hline & $\mathrm{ICN}$ & Bandwidth and power saving & o & o & o & + & o & o & o \\
\hline
\end{tabular}

channel.

\section{B. Physical layer features}

Satellite systems usually operate in frequency bands ranging from 1 to $40 \mathrm{GHz}$ [9]. Low-frequency bands such as the L-band $(1-2 \mathrm{GHz})$ and the S-band $(2-4 \mathrm{GHz})$ need lower pointing accuracy and antenna gain compared to higher frequency bands such as Ku-band (12-18 GHz), K-band (18$27 \mathrm{GHz})$ and Ka-band (27-40 GHz), which however usually guarantee higher data rates. For this reason, they should be preferred for SG applications with moderate and high mobility requirements. Also, high frequency bands are susceptible to weather conditions thus impacting availability. In particular, $\mathrm{Ku}$ and $\mathrm{Ka}$ bands availability ranges from $99.7 \%$ to $99.9 \%$ due to the possibility of heavy rains, snowstorms and thick cloud cover. Differently, the L-band ensures reliability up to 99.99\% thanks to the resilience to weather conditions. If the considered SG application requires higher availability (over $99.99 \%$ ), than the use of a dual band system is necessary. This is the case for safe-critical applications such as T\&D monitoring and distribution automation, which could use the L-band to backup the primary band (e.g., Ku or Ka), so that the overall availability is increased to more than $99.9999 \%$. Table II summarizes these needs for the SG applications considered.

As to the domain of modulation and coding, in the last years important improvements have been achieved in the mitigation of the sensitivity to rain fade. The most important is the use of Adaptive Coding and Modulation (ACM) techniques, which guarantee a satisfying reliability even during inclement weather conditions at the cost of a reduced data rate [10]. This technology represents an interesting choice for applications involving video and audio, such as mobile workforce and video surveillance.

\section{Channel Resource Management}

One of the most active research fields in SATCOM deals with channel resource access. Several Medium Access Channel (MAC) techniques have been recently improved to satisfy the unique characteristics of SATCOM and M2M applications, as summarized in Table III.

1) Fixed Allocation (FA): The easiest way to grant channel access is by permanently allocating a fixed amount of channel resource to the involved users using either Time Division Multiple Access (TDMA), Frequency Division Multiple Access (FDMA) or Code Division Multiple Access (CDMA). This solution enables tight control on the delay and complete availability from the data link viewpoint. Thus, it is ideal for SG applications needing frequent data transmission such as video surveillance, but also with high requirements on latency and availability as T\&D monitoring. Depending on the criticality of the application, it could be used also with variable data traffic as in the case of remote substations and distribution automation. Nevertheless, when the expected traffic is variable, the channel use is less efficient.

2) Demand Assignment Multiple Access (DAMA): DAMA protocols improve poor link utilization in case of variable traffic by means of dynamic channel allocation. To do so, a Network Control Center (NCC) is used. Reservation of 
Table III: MAC protocols and characteristics

\begin{tabular}{|c|c|c|c|c|c|c|}
\hline Category & Allocation & Contention & Traffic & Advantages & Disadvantages & Protocols \\
\hline FA & Static & Free & $\begin{array}{c}\text {-Periodic and } \\
\text { deterministic } \\
\text { •High data rates possible }\end{array}$ & $\begin{array}{l}\text {-Strong QoS guarantee } \\
\text { •Easy to implement } \\
\text { •NCC not needed } \\
\text { •Low latency }\end{array}$ & $\begin{array}{l}\text {-Inefficient for } \\
\text { variable or } \\
\text { infrequent traffic }\end{array}$ & $\begin{array}{l}\text { TDMA } \\
\text { FDMA } \\
\text { CDMA }\end{array}$ \\
\hline DAMA & Dynamic & $\begin{array}{l}\text { It depends } \\
\text { on the } \\
\text { protocol }\end{array}$ & $\begin{array}{l}\text {-Variable or infrequent } \\
\text {-High data rates possible }\end{array}$ & $\begin{array}{c}\text {-Efficient for variable traffic } \\
\text { and data rates } \\
\text { •Fairness possible }\end{array}$ & $\begin{array}{l}\text { •High latency on } \\
\text { resource allocation } \\
\text { request }\end{array}$ & $\begin{array}{c}\text { PRMA } \\
\text { CF-DAMA }\end{array}$ \\
\hline RA & Dynamic & Yes & $\begin{array}{c}\text { •Bursty } \\
\text { •Best effort with } \\
\text { unassigned data rate } \\
\text {-Real time without QoS } \\
\text { guarantee }\end{array}$ & $\begin{array}{l}\text { - Low latency and high } \\
\text { efficiency in light traffic } \\
\text {-Simple to implement }\end{array}$ & $\begin{array}{l}\text {-Low efficiency and } \\
\text { high latency in } \\
\text { heavy traffic } \\
\text { •No QoS guarantee }\end{array}$ & $\begin{array}{l}\text { ALOHA } \\
\text { CRDSA } \\
\text { CSA }\end{array}$ \\
\hline
\end{tabular}

the channel resource can be granted using contention as in Packet Reservation Media Access (PRMA) or exploiting the unused capacity that the NCC allocates to active terminals as in Combined Free-DAMA (CF-DAMA). High data rates are possible with DAMA, but depending on the reservation policy adopted they could not be guaranteed for fairness reasons among terminals. Moreover, the reservation delay equals at least the satellite RTT and can thus be too high for some SG applications, especially if bent-pipe GEO satellites are used. DAMA is convenient both in case of variable traffic or infrequent traffic with significant bandwidth requirements, but the delay allowed by the considered SG application to reserve the necessary capacity should be taken into account. Therefore, examples of applications that can exploit DAMA include mobile workforce and AMI backhaul.

3) Random Access (RA): In RA, users contend for using the data channel without any NCC coordination. The most wellknown RA protocol is Aloha. In the last years, the performance of these RA protocols has been improved thanks to the use of interference cancellation to restore colliding packets, as in Contention Resolution Diversity Slotted Aloha (CRDSA) [11]. A further improvement is Coded Slotted Aloha (CSA), which combines interference cancellation with packet erasure correcting codes. RA is simple to implement on the sender side. Moreover, it is ideal for infrequent but also variable traffic with loose requirements on availability, bandwidth and latency. Nevertheless, low delay and high throughput can also be obtained in case of light channel load, although the service provided is best effort. As the traffic increases, RA protocols decrease in performance both in terms of latency, bandwidth and availability. For this reason, the most indicated applications using RA are remote generation plants and EVs.

\section{Local intelligence}

Considering the pace at which intelligent devices are added to the electric grid and the resulting overload on the communication infrastructure, many applications are shifting towards variable or bursty activity modes. To do so, local intelligence is exploited to transmit only insightful information based on the actual need. To this, [12] proposes that the same predictive model of power generation is installed at both the power generation site and the remote utility control center, so that only measurements which are significantly different from the expected ones are sent. The same approach can be applied also to SMs. Similarly, in [13] only insightful PMU measurements are sent to the remote T\&D monitoring center. The rules for data transmission can vary depending on the needs and can be modified at runtime from the remote control center. The use of the same paradigm is also foreseen by next-generation cameras, which leverage on the intelligence of the camera itself to parse acquired video frames and reveal possible risks and threats to be communicated. The implementation of these concepts would diminish the resource needs of SG applications although at the cost of increased traffic variability and fuzziness.

These concepts of local intelligence can also be used for inward communications, such as the case of commands sent to field devices as in remote substation and distribution automation. As a matter of fact, some protection functions have really strict latency constraints that require immediate action (i.e., within few ms) [5] [6]. SATCOM are not able to satisfy such a requirement. Nevertheless, for those scenarios in which alternative ways of communication are not possible, SATCOM can serve as a channel to install, uninstall and modify protection rules at the local site, to satisfy the requirements of the considered application.

\section{E. Open networking and transport protocols}

Although in current satellite systems most network and transport protocols are proprietary, other initiatives such as 3GPP long-term evolution (LTE) over satellite [14] and the second generation of DVB standards are trying to offer some open alternatives [15]. While the former has a relevant role for mobile workforce, the latest is important because supports IPv6 using Generic Stream Encapsulation (GSE). Not only the use of IPv6 is compliant with the set of protocols envisioned for M2M, but it is also important to guarantee interoperability in heterogeneous networks.

Another novel paradigm at this level is Information-Centric Networking (ICN), which relies on routing information by 
content rather than by host. Several ICN approaches are currently being proposed. The publish-subscribe paradigm is probably the most well-known. As proposed in [16], the ICN paradigm can be applied to satellite networks, yielding to bandwidth and power savings as well as to a reduced signaling congestion by broadcasting or multicasting the same message to a huge number of earth terminals under the same footprint. Considering that some SG applications foresee command delivery to a group of actuators, this represents a natural application scenario for group-based communications as in the case of remote substation and distribution automation.

\section{Conclusions}

This paper has provided an overview on the role satellite communications can play in different smart grid scenarios. The use of satellite solutions is becoming more and more economically feasible, as the cost of the satellite equipment and the prices of the relevant services are continuously decreasing. In addition, satellite communication services are distanceinsensitive and scale well with the increase of the area to cover, making them attractive for deployments over wide areas. In the future, it is expected that more and more SG applications leveraging SATCOM will emerge, conquering a relevant role in the deployment of smart grid services. To do so, it is of utmost importance to understand the available technologies and the ongoing challenges to achieve the requirements of the considered domain. As it has been shown, these requirements are theoretically reachable, but further research is needed to implement strategies specifically optimized for the smart grid domain especially in LEO systems, which are envisioned to take the biggest share.

Applications requiring high throughput and low latency communications will likely be supported by those systems designed to support voice connectivity. On the other hand, current satellites are not adequate for very large populations of devices with different bandwidth and energy constraints as M2M applications show. This is the area in which research efforts are likely to concentrate more. It is not a case, that the European Space Agency has recently launched an initiative through its Advanced Research in Telecommunications Systems program to encourage SATCOM prototypes for M2M applications.

\section{LIST OF ABBREVIATIONS}

\begin{tabular}{l|l} 
ACM & Adaptive Coding and Modulation \\
AMI & Advanced Metering Infrastructure \\
BGAN & Broadband Global Area Network \\
CDMA & Code Division Multiple Access \\
CF-DAMA & Combined Free-DAMA \\
CRDSA & Contention Resolution Diversity Slotted Aloha \\
CSA & Coded Slotted Aloha \\
DAMA & Demand Assignment Multiple Access \\
DER & Distributed Energy Resources \\
EV & Electric Vehicle
\end{tabular}

\begin{tabular}{l|l} 
FA & Fixed Allocation \\
FDMA & Frequency Division Multiple Access \\
GEO & Geostationary Earth Orbit \\
GSE & Generic Stream Encapsulation \\
ICN & Information-Centric Networking \\
LEO & Low Earth Orbit \\
LoRaWAN & Long Range Wide Area Network \\
LTE & Long Term Evolution \\
MAC & Medium Access Channel \\
MEO & Medium Earth Orbit \\
M2M & Machine-to-Machine \\
NCC & Network Control Center \\
PMU & Phasor Measurement Unit \\
PRMA & Packet Reservation Media Access \\
QoS & Quality of Service \\
RA & Random Access \\
RSU & Road Side Unit \\
RTT & Round Trip Time \\
SATCOM & Satellite Communications \\
SCADA & Supervisory Control And Data Acquisition \\
SG & Smart Grid \\
SM & Smart Meter \\
TDMA & Time Division Multiple Access \\
T\&D & Transmission and Distribution \\
&
\end{tabular}

\section{ACKNOWLEDGEMENT}

This work has been supported by Regione Autonoma della Sardegna, L.R. 7/2007: "Promozione della ricerca scientifica e dell'innovazione tecnologica in Sardegna, annualità 2012, CRP-60511".

\section{REFERENCES}

[1] B. Gohn and C. Wheelock, "Smart grid network technologies and the role of satellite communications," Pike Research LLC, Boulder, CO, 2010.

[2] M. D. Sanctis, E. Cianca, G. Araniti, I. Bisio, and R. Prasad, "Satellite communications supporting internet of remote things," IEEE Internet of Things Journal, vol. 3, no. 1, pp. 113-123, Feb 2016.

[3] G. Cocco and C. Ibars, "On the feasibility of satellite $\mathrm{m} 2 \mathrm{~m}$ systems," in AIAA Intl Comm. Satellite Systems Conference (ICSSC), 2012.

[4] Q. Yang, D. I. Laurenson, and J. A. Barria, "On the use of LEO satellite constellation for active network management in power distribution networks," IEEE Transactions on Smart Grid, vol. 3, no. 3, pp. 13711381, Sept 2012.

[5] IEC61850-5 Edition, "Communication networks and systems in substations - Part 5: Communication requirements for functions and device models."

[6] V. C. Gungor, D. Sahin, T. Kocak, S. Ergut, C. Buccella, C. Cecati, and G. P. Hancke, "A survey on smart grid potential applications and communication requirements," IEEE Transactions on Industrial Informatics, vol. 9, no. 1, pp. 28-42, Feb 2013.

[7] R. H. Khan and J. Y. Khan, "A comprehensive review of the application characteristics and traffic requirements of a smart grid communications network," Computer Networks, vol. 57, no. 3, pp. 825 - 845, 2013.

[8] C. Wietfeld and C. Ide, "Vehicle-to-infrastructure communications," Vehicular Communications and Networks: Architectures, Protocols, Operation and Deployment, 2015.

[9] J. N. Pelton, S. Madry, and S. Camacho-Lara, Handbook of satellite applications. Springer Publishing Company, Incorporated, 2012.

[10] E. Alberty, S. Defever, C. Moreau, R. D. Gaudenzi, A. Ginesi, R. Rinaldo, G. Gallinaro, and A. Vernucci, "Adaptive coding and modulation for the dvb-s2 standard interactive applications: Capacity assessment and key system issues," IEEE Wireless Communications, vol. 14, no. 4, pp. 61-69, August 2007. 
[11] O. D. R. Herrero and R. D. Gaudenzi, "High efficiency satellite multiple access scheme for machine-to-machine communications," IEEE Transactions on Aerospace and Electronic Systems, vol. 48, no. 4, pp. 2961-2989, October 2012.

[12] T. Kleinschmidt and C. Wietfeld, "Fluctuation-sensitive modelpredictive communication for distributed energy resources," in IEEE ICC 2016 Workshop on Integrating Communications, Control, and Computing Technologies for Smart Grid, May 2016.

[13] A. Meloni, P. A. Pegoraro, A. Atzori, P. Castello, and S. Sulis, "Iot cloud-based distribution system state estimation: Virtual objects and context-awareness," in 2016 IEEE International Conference on Communications (ICC), May 2016, pp. 1-6.

[14] M. Papaleo, M. Neri, A. Vanelli-Coralli, and G. E. Corazza, "Using lte in 4G satellite communications: Increasing time diversity through forced retransmission," in 2008 10th International Workshop on Signal Processing for Space Communications, Oct 2008, pp. 1-4.

[15] ETSI EN 301-545-2, Digital video broadcasting (DVB); second generation DVB interactive satellite system (DVB-RCS2); part 2: lower layers for satellite standard, 2012.

[16] A. Detti, A. Caponi, and N. Blefari-Melazzi, "Exploitation of information centric networking principles in satellite networks," in 2012 IEEE First AESS European Conference on Satellite Telecommunications (ESTEL), Oct 2012, pp. 1-6.

Alessio Meloni (S'12,M'15) received his Ph.D. degree from the University of Cagliari (Italy) in March 2014. Since 2011, he has been with the Multimedia and Communications Lab - University of Cagliari. He has been guest $\mathrm{PhD}$ student at the German Aerospace Center in Oberpfaffenhofen. His research interests include wireless communications, Internet of Things and Smart Grids. He is a member of IEEE, IEEE ComSoc, CNIT and IEEE Smart GridTAC.

Luigi Atzori (SM'09) is Associate Professor at the Department of Electrical and Electronic Engineering at the University of Cagliari (Italy), where he leads the laboratory of Multimedia and Communications. L. Atzori research interests are in multimedia communications and computer networking (wireless and wireline), with emphasis on multimedia QoE, multimedia streaming, NGN service management, service management in wireless sensor networks, architecture and services in the Internet of Things. 